\title{
RAFT Macro-Surfmers and Their Use in the ab Initio RAFT Emulsion Polymerization To Decouple Nanoparticle Size and Polymer Molecular Weight
}

\author{
Umberto Capasso Palmiero, ${ }^{\dagger}$ Azzurra Agostini, ${ }^{\dagger}$ Simone Gatti, ${ }^{\dagger}$ Mattia Sponchioni, $^{\dagger}$ Viola Valenti, $^{\dagger}$ \\ Lucia Brunel, ${ }^{\dagger}$ and Davide Moscatelli*, ${ }^{+\dagger}$ \\ ${ }^{\dagger}$ Department of Chemistry, Materials and Chemical Engineering, Politecnico di Milano, Via Mancinelli 7, 20131 Milano, Italy \\ ${ }^{\ddagger}$ Department of Chemical and Biological Engineering, Northwestern University, Evanston, Illinois 60208, United States
}

\section{Supporting Information}

ABSTRACT: Polymeric nanoparticles (NPs) are highly engineered nanoemulsions with applications in several fields. The control over NP surface chemistry, size (Dn), and molecular weight (MW) of the polymer they are made up of plays a paramount role in the optimization of their end-use performance. In this work, the theoretical basis to decouple the dependence between the NP Dn and MW has been presented, and an operative way has been demonstrated via $\mathrm{ab}$ initio reversible addition-fragmentation chain transfer (RAFT) emulsion polymerization (AIREP), a "living" heterogeneous process that adopts RAFT macro-surfmers: macromolecular chain transfer agents (CTA) produced via RAFT polymerization of amphiliphic monomers, such as surfmers. The possibility of obtaining the desired length of the lipophilic block or the length of the whole block copolymer and the NP Dn by choosing the correct length of the RAFT macro-surfmer has been assessed. It has been discovered that a wide range of Dn and MW can be achieved, but not very big NPs with very low copolymer MW; this limit is consistent with the physical and geometric constraints of the system.

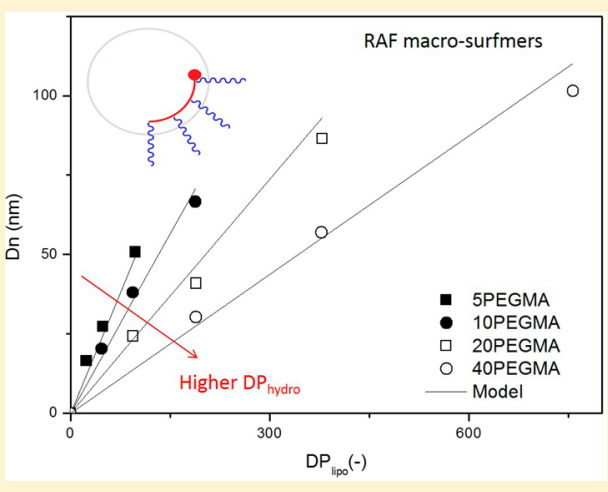

\section{INTRODUCTION}

In the recent years, polymeric nanoparticles (NPs) have gained significant attention in several fields such as waterborne coatings, adhesives, and medicine. Consequently, literature about this topic is growing, and new methods have been developed to improve the NP performance through the finetuning of paramount properties such as NP surface chemistry, size (Dn), and molecular weight (MW) of the polymer that they are made up of. ${ }^{1-4}$ As an example, waterborne latexes have been adopted to produce environmentally friendly coatings, and the latex Dn and MW affect the coalescence mechanism and formation of the final polymeric film and, thus, its mechanical properties. ${ }^{5,6}$ In a similar field, where the latexes are used to produce pressure-sensitive adhesives, it is also wellknown that smaller NPs and high MW acrylic polymers are responsible for the improvement of the adhesive properties. ${ }^{7}$ In medicine, NPs have been used as carriers for the delivery of various therapeutics, such as anticancer drugs and small interfering RNA (siRNA). ${ }^{8}$ In this application, Dn and surface chemistry play important roles as they affect the therapeutics' biodistribution and uptake when injected via IV bolus while polymer MW determines the accumulation into the body and, thus, their toxicity. 9,10

According to the literature, ${ }^{11,12}$ one of the most effective techniques used to synthesize NPs is emulsion polymerization (EP) due to the fact that (i) it does not require toxic cosolvent and expensive postprocesses to remove it, (ii) it favors high reaction rates due to the radical compartmentalization, (iii) it allows fast heat dissipation and production of high concentrated latexes, and (iv) it can be easily translated in industry. EP allows for the direct synthesis of a latex through a heterogeneous process: a slightly soluble monomer, a surfactant, and an initiator are dissolved in water and heated at high temperature for several hours. In addition, it is possible to control the NP Dn by varying the monomer-to-surfactant ratio and/or adopting a different monomer feeding procedure, such as monomer starved semibatch emulsion polymerization (MSSEP). ${ }^{13}$ However, some drawbacks such as the need of toxic surfactants to maintain colloidal stability ${ }^{14,15}$ and the difficulties related to the control over the MW limit its application when the fine-tuning of these properties are highly required. In fact, emulsion polymerization is a free radical polymerization process carried out in an heterogeneous phase, and thus, the length of the produced chains is affected by the kinetic constants, such as the propagation and termination constants, and not only by the monomer and initiator concentrations throughout the reaction. ${ }^{11,12}$ However, the development of the so-called controlled living polymerization

Received: August 25, 2016

Revised: November 3, 2016

Published: November 11, 2016 
Table 1. Characterization of the PEGylated Macro-CTA

\begin{tabular}{|c|c|c|c|c|c|c|c|c|}
\hline sample & time $[\mathrm{h}]$ & $\chi_{\mathrm{GPC}}$ & $\chi_{\text {NMR }}$ & $M_{\mathrm{n}, \mathrm{GPC}}[\mathrm{Da}]$ & $M_{\mathrm{n}, \mathrm{NMR}}[\mathrm{Da}]$ & $M_{\mathrm{n}, \text { Theory }}[\mathrm{Da}]$ & $\mathrm{DP}_{\text {hydro }}$ & $Ð$ \\
\hline 5PEGMA & 24 & 92.8 & 91.0 & 6957 & 4910 & 4492 & 4.63 & 1.09 \\
\hline 10PEGMA & 24 & 92.1 & 90.5 & 9141 & 9420 & 8556 & 9.14 & 1.07 \\
\hline 20PEGMA & 24 & 90.8 & 89.0 & 12025 & 18970 & 16906 & 18.69 & 1.09 \\
\hline 40PEGMA & 24 & 90.9 & 89.3 & 18531 & 37870 & 33847 & 37.59 & 1.09 \\
\hline
\end{tabular}

(CRP), such as nitroxide-mediated radical polymerization (NMP), ${ }^{16}$ atom transfer radical polymerization (ATRP), ${ }^{17}$ and reversible-addition chain transfer polymerization (RAFT) ${ }^{18}$ have improved the possibility of obtaining the desired MW through the addition of a "living agent" and, thus, by varying the molar ratio between this agent and the monomer. Among all of the techniques adopted, the RAFT polymerization can be applied to a wide variety of monomers at temperatures comparable to those of the conventional radical polymerization. $^{19,20}$ In addition, since RAFT is based on a degenerative mechanism, where the livingness is assured by the fast exchange reactions between the propagating radicals and the chain transfer agent, it is one of the few controlled radical polymerization that can be efficiently translated in a heterogeneous polymerization. ${ }^{21}$

Since the chain transfer agent, a thio compound, ideally remains attached to each polymer chain at the end of the reaction, ${ }^{18}$ it can be used to restart the growth of these "dormant" polymers by further addition of the same or of a different monomer opening to the production of well-defined block copolymers. ${ }^{22}$ Ferguson et al. ${ }^{23}$ used these RAFT characteristics to produce amphiphilic species able to act in the EP both as surfactants and MW controlling agents. This ab initio RAFT emulsion polymerization (AIREP) does not require toxic surfactants and has opened the possibility of producing NPs with different MWs, surface functionalizations, and morphologies. ${ }^{24}$ In contrast to NPs obtained via EP, the core and the shell are covalently attached and correspond to the two polymer segments of the constituting block copolymer.

However, to the best of our knowledge, there are no works devoted to the study of the relationship between the Dn, MW, and the living character of the RAFT polymerization, and only one attempt has been published to tune independently NP Dn and MW by adopting a controlled radical polymerization. ${ }^{25}$ Sebahky et $\mathrm{al}^{25}$ adopted a brilliant temperature responsive nanoreactor technology based on a poly(dimethylacrylamide)$b$-poly $(N$-isopropylacrylamide) $\mathrm{p}($ MDA- $b$-NIPAM $)$ surfactant. This block copolymer acts as a "nanoparticle assembler", producing micelles at a temperature above the lower critical solution temperature (LCST) of the pNIPAM block. In order to control the MW of the polymer, a macromolecular RAFT agent made of several NIPAM units is added with the hydrophobic monomer to the reaction. After the completion of the polymerization, the system is cooled below the LCST of pNIPAM, and the NPs made of the block copolymer PNIPAM$b$-PSt are released by the nanoreactors. The control over the nanoreactor size allows for the tuning of the Dn of the final NP while the control over the ratio between the hydrophobic monomer (styrene in this work) and of the macromolecular RAFT agent leads to obtain the desired MW independently. However, in this case the RAFT end group is not directly attached to the stabilizing species, and a nonreactive temperature responsive surfactant is required to maintain colloidal stability. Indeed, the decoupling is related to a correct distribution of the controlling species into all the growing NPs.
In this work, the theoretical conditions required to decouple the NP Dn and MW in the ab initio RAFT emulsion polymerization without the need of any nonreactive surfactant have been developed. It has been found that this is possible by adopting RAFT macro-surfmers: RAFT macromolecular amphiphilic species produced by the RAFT polymerization of a surfmer. Their use has allowed for the substitution of the hydrophilic chains of the final block copolymer formed via AIREP with amphiphilic chains.

Four RAFT macro-surfmers with different MW have been synthesized via the RAFT polymerization of a PEGylated methacrylate (PEGMA), and their CMCs have been evaluated by the pyrene fluorescence technique in order to show that they still have an amphiphilic behavior after RAFT polymerization and that the CMC are quite similar. Then they have been used in the AIREP of MMA. The final results showed that the ability of the macro-RAFT surfmers to cover the surface of the NP (surface coverage $A_{\mathrm{Cov}}\left(\mathrm{nm}^{2}\right)$ ) is a key parameter and a linear function of their length for this class of RAFT macro-surfmers. This dependence has been fitted and used to simulate the experimental data. Simulations have shown the possibility of obtaining the desired Dn and MW by choosing the correct length of the amphiphilic block. Interestingly, it has been discovered that it is not possible to obtain very big NPs with very low copolymer MW. This is in agreement with the physical and geometric limits of the system.

\section{MATERIALS AND METHODS}

Materials. Poly(ethylene glycol) methyl ether methacrylate (PEGMA, $M_{\mathrm{n}} 950 \mathrm{Da}$, Sigma-Aldrich), 4,4'-azobis(cyanovaleric acid) (ACVA, 98\%, Sigma-Aldrich), 4-cyano-4-(phenylcarbonothioylthio)pentanoic acid (CPA, >97\%, Sigma-Aldrich), methyl methacrylate (MMA, 99\%, Sigma-Aldrich), and pyrene (99\%, Sigma-Aldrich) were used as received except when specifically noted. All the solvents used were of analytical-grade purity and were purchased from SigmaAldrich.

Synthesis and Characterization of PEGylated RAFT MacroSurfmers. The PEG-based RAFT macro-surfmers with different MWs were synthesized via RAFT polymerization of PEGMA varying the degree of polymerization according to

$$
\mathrm{MW}_{\mathrm{b} \text {,hydro }}=\mathrm{MW}_{\text {hydro }} \mathrm{DP}_{\text {hydro }}+\mathrm{MW}_{\text {RAFT agent }}
$$

where $M_{\mathrm{b}, \text { hydro }}$ is the desired molecular weight of the final polymer, $\mathrm{DP}_{\text {hydro }}$ is the degree of polymerization, and $\mathrm{MW}_{\text {hydro }}$ and $\mathrm{MW}_{\mathrm{RAFT} \text { agent }}$ are the molecular weight of the PEGMA and of the CPA, respectively.

Four different $\mathrm{DP}_{\text {hydro }}$ equal to $5,10,20$, and 40 were chosen at a monomer concentration of $20 \mathrm{wt} \%$ and with an initiator to RAFT agent ratio equal to $1 / 3$. As example, in the first case (sample named 5PEGMA in Table 1) $3.94 \mathrm{~g}$ (4.15 mmol) of PEGMA, $232 \mathrm{mg}(0.82$ $\mathrm{mmol})$ of CPA, and $70 \mathrm{mg}(0.24 \mathrm{mmol})$ of ACVA were dissolved in $20 \mathrm{~mL}$ of ethanol. The solution was poured in a septum-sealed flask, purged for $10 \mathrm{~min}$ with nitrogen, and heated to $65^{\circ} \mathrm{C}$ in a constant temperature oil bath under magnetic stirring. In order to evaluate the kinetics of the RAFT polymerization, aliquots of the mixtures were taken after 2, 4, 6, 8, and $24 \mathrm{~h}$ and dried under nitrogen. $200 \mathrm{mg}$ of the final dried mixture was then dissolved in $20 \mathrm{~mL}$ of water and purified two times via ultrafiltration by Vivaspin 20 (Sartorius, 3 or $10 \mathrm{kDa}$ cutoff) in order to remove the unreacted monomer. 
The collected samples were characterized by ${ }^{1} \mathrm{H}$ NMR and gel permeation chromatography (GPC).

For the NMR, about $10 \mathrm{mg}$ of dried polymer was dissolved in 0.6 $\mathrm{mL}$ of $\mathrm{CDCl}_{3}$ and analyzed by a $400 \mathrm{MHz}$ apparatus (Bruker, Switzerland).

The conversion and the degree of polymerization $\left(\mathrm{DP}_{\text {hydro }}\right)$ were calculated by the following equations:

$$
\begin{aligned}
& X_{\mathrm{NMR}}=1-\frac{3 A_{d}}{A_{h}} \\
& \mathrm{DP}_{\text {hydro }}=1-\frac{2 X A_{h}}{3 A_{a}}
\end{aligned}
$$

where $X_{\mathrm{NMR}}$ is the monomer conversion, and $A_{a}$ and $A_{h}$ are the areas of the peaks $a$ and $h$ in the NMR spectrum reported in Figure S1 (Supporting Information). The molecular weight of the polymer $\left(M_{n, N M R}\right)$ can be then calculated via eq 1 .

For the GPC, samples were dissolved in THF at a concentration of $4 \mathrm{mg} \mathrm{mL}^{-1}$ after filtration through a $0.45 \mu \mathrm{m}$ pore-size membrane and analyzed with a Jasco (Series) apparatus. The separation was performed at a flow rate of $0.5 \mathrm{~mL} \mathrm{~min}{ }^{-1}$ and at $35{ }^{\circ} \mathrm{C}$ with three Superchrom PLgel $5 \mu \mathrm{m}$ columns $(600 \times 7.5 \mathrm{~mm}$, measuring range $0.5-1000 \mathrm{kDa}$ ).

$M_{\mathrm{n}, \mathrm{GPC}}$ and dispersity $(\nexists)$ were determined from differential refractive index (RI) data and were relative to poly(styrene) standards (from 580 to $197300 \mathrm{~g} / \mathrm{mol}$, Polymer Laboratories). Conversion was determined according to

$$
X_{\mathrm{GPC}}=\frac{A_{\mathrm{pol}}}{A_{\mathrm{pol}}+A_{\mathrm{mon}}}
$$

where $A_{\text {pol }}$ and $A_{\text {mon }}$ are respectively the area under the RI signal curve of polymer and monomer, as visible in Figure S2 (Supporting Information).

Equation 4 is true under the assumption that the refractive index increment $(\mathrm{d} n / \mathrm{d} c)$ of the polymer and of the monomer are equal or very similar.

In fact, the area under curve in a RI detector is equal to

$$
A=K_{\mathrm{d}} \frac{\mathrm{d} n}{\mathrm{~d} c} m_{\text {inj }}
$$

where $K_{\mathrm{d}}$ is an instrument constant and $m_{\text {inj }}$ is the injected mass of the species analyzed.

Substituting the eq 5 into the conversion definition, it is possible to obtain the above-mentioned eq 4 without the approximation of the same $\mathrm{d} n / \mathrm{d} c$ :

$$
X_{\mathrm{GPC}}=\frac{m_{\mathrm{pol}}^{\mathrm{inj}}}{m_{\mathrm{pol}}^{\text {inj }}+m_{\mathrm{mon}}^{\text {inj }}}=\frac{A_{\mathrm{pol}} / \mathrm{d} n / \mathrm{d} c_{\mathrm{pol}}}{A_{\mathrm{pol}} / \mathrm{d} n / \mathrm{d} c_{\mathrm{pol}}+A_{\mathrm{mon}} / \mathrm{d} n / \mathrm{d} c_{\text {mon }}}
$$

CMC Determination of PEGMA and RAFT Macro-Surfmers. CMC of both PEGMA monomer and PEG-based RAFT macrosurfmers was evaluated via spectrofluorometry using pyrene as fluorescent probe. A predetermined amount of pyrene in acetone was transferred into different scintillating vials, and the acetone was allowed to evaporate at $40^{\circ} \mathrm{C}$ for $1 \mathrm{~h}$. A series of aqueous solutions of each sample were prepared with concentrations ranging from 0.001 to $100 \mathrm{mg} / \mathrm{mL}$ and then added to the vials. In each vial the polymer aqueous solution was added with such a volume that the final concentration of pyrene was equal to $6 \times 10^{-7} \mathrm{M}$. The system was vigorously mixed using a vortex (IKA Vortex Genius) and allowed to equilibrate for $24 \mathrm{~h}$ while avoiding a direct exposure to light. Then, fluorescence emission spectra were recorded on a Jasco FP8500 spectrofluorometer. Excitation was carried out at $335 \mathrm{~nm}$. The emission spectra were recorded for a range of $350-450 \mathrm{~nm}$. Excitation and emission band widths were set at 5 and $2 \mathrm{~nm}$, respectively. To determine the CMC, the intensity ratio of the third band (384 nm, $I_{3}$ ) to the first band $\left(373 \mathrm{~nm}, I_{1}\right)$ of the pyrene emission spectra was studied as a function of the sample concentration.
Ab Initio RAFT Emulsion Polymerization of MMA. The RAFT macro-surfmers previously synthesized were used to produce NPs via ab initio RAFT emulsion polymerization of MMA. In this case the DP expressed in eq 1 is the number of the units of MMA attached to the final copolymer $\left(\mathrm{DP}_{\text {lipo }}\right)$. In order to better examine the behavior of the system by varying the $\mathrm{DP}_{\text {lipo }}$ and $\mathrm{DP}_{\text {hydro }}$ parameters, a $\mathrm{DP}_{\text {ratio }}$ was defined as

$$
\mathrm{DP}_{\text {ratio }}=\frac{\mathrm{DP}_{\text {lipo }}}{\mathrm{DP}_{\text {hydro }}}
$$

In all the experiments the weight concentration of the RAFT macrosurfmers and the initiator to RAFT macro-surfmers molar ratio were taken constant to $50 \mathrm{mg} / \mathrm{mL}$ and to $1 / 2$, respectively. Three different MMA concentrations were used in order to obtain $\mathrm{DP}_{\text {ratio }}=5,10$, and 20. In a typical experiment (e.g., P5-1 in Table 3), $0.15 \mathrm{~g}\left(3.05 \times 10^{-5}\right.$ $\mathrm{mol})$ of 5PEGMA and $4 \mathrm{mg}\left(1.43 \times 10^{-5} \mathrm{~mol}\right)$ of ACVA were dissolved in $3 \mathrm{~mL}$ of an aqueous acetic buffer $\left(\mathrm{pH}=5.2,0.27 \mathrm{~mol} \mathrm{~L}^{-1}\right.$ acetic acid, and $0.73 \mathrm{~mol} \mathrm{~L}^{-1}$ ) using a $4 \mathrm{~mL}$ vial. This mixture was stirred to obtain a homogeneous solution. Then, $75 \mathrm{mg}\left(7.49 \times 10^{-4}\right.$ mol) of MMA was added. The sealed vial was purged with nitrogen for 2 min and immersed in a constant temperature oil bath at $65^{\circ} \mathrm{C}$ and at a stirring rate of $400 \mathrm{rpm}$. After $24 \mathrm{~h}$, the latex was characterized by dynamic laser light scattering analysis (DLLS, Zetasizer Nano Series, Malvern Instruments) and transmission electron microscopy (TEM). TEM was performed using a Philips CM200 electron microscope operating at $200 \mathrm{kV}$ equipped with a field emission gun filament. The NP suspensions were dropped onto a 200 mesh carbon-coated copper grid and air-dried for several hours before analysis. A Gatan US 1000 CCD camera was used, and $2048 \times 2048$ pixels images with 256 gray levels were recorded. In addition, an aliquot of the latex was dried under air, and the MW of the final copolymer was evaluated via GPC as already described for the characterization of the RAFT macrosurfmers. MMA conversion was determined by gravimetry. In the case of experiment P5-3 in Table 3, aliquots of the mixture were taken after $1,2,4$, and $8 \mathrm{~h}$ to study the kinetic of the polymerization.

\section{RESULTS AND DISCUSSION}

In this work, a conventional ab initio RAFT emulsion polymerization was studied in which the NP are formed only by amphiphilic block copolymers. According to the RAFT living characteristics, the MW of the block copolymer that forms the NP can be expressed as

$$
\mathrm{MW}_{\text {cop }}=\mathrm{MW}_{\text {hydro }} \mathrm{DP}_{\text {hydro }}+\mathrm{MW}_{\text {lipo }} \mathrm{DP}_{\text {lipo }}+\mathrm{MW}_{\mathrm{RAFT} \text { agent }}
$$

where $\mathrm{MW}_{\text {hydro }}, \mathrm{MW}_{\text {lipo, }}$ and $\mathrm{MW}_{\mathrm{RAFT} \text { agent }}$ are the molecular weights of the hydrophilic monomer, the lipophilic monomer, and the RAFT agent adopted in the process. $\mathrm{DP}_{\text {hydro }}$ and $\mathrm{DP}_{\text {lipo }}$ are the degrees of polymerization of the hydrophilic and lipophilic block and are equal to the molar concentration of the hydrophilic monomer to the RAFT agent and the lipophilic monomer to the RAFT agent ratio, respectively.

Equation 8 describes the number of units attached to each block of a binary system and represents the final product of the two or three subsequent RAFT polymerization steps necessary to obtain the final latex. Two steps are required in the case of the polymerization-induced self-assembly ( PISA $^{26,27}$ ) technique that consists of (i) a RAFT polymerization of a water-soluble monomer to produce the hydrophilic part and (ii) growth of the second block copolymer and consequent self-assembly into water to form NPs via RAFT polymerization of a lipophilic monomer. Otherwise, the classic ab initio RAFT emulsion polymerization process may require three RAFT reactions: (i) production of the hydrophilic block, (ii) addition to enough lipophilic units to make the copolymer a surface active species, and (iii) further growth of the lipophilic block in water to 
produce NPs. ${ }^{23,28-32}$ In this latter case, the number of monomer units attached to the lipophilic block $\left(\mathrm{MW}_{\text {lipo }} \cdot \mathrm{DP}_{\text {lipo }}\right.$ in eq 8) is equal to the sum of the units added in the last two RAFT polymerizations.

Since the produced block copolymer are expected to be highly ordered, we can consider for each case that (i) the lipophilic block of the copolymer are preferentially located inside the core and (ii) the hydrophilic part is almost completely confined on the NP surface. This implies that the charge burial is negligible, which is reasonable if we consider that these are big species and that they are not randomly incorporated into the backbone as classic surfmers. ${ }^{33}$ Indeed, we expect that the ability of the hydrophilic block to cover and stabilize the surface will be a paramount parameter that influences the Dn and the MW that can obtained and also influences the NP morphology. ${ }^{24}$ For this reason, the surface area that a single hydrophilic chain can cover has been evaluated as the ratio between the surface area of a single NP and the total number of hydrophilic chains located on the NP surface $\left(N_{\mathrm{b}, \text { hydro }}\right)$ according to ${ }^{34}$

$$
A_{\mathrm{Cov}}=\frac{\pi D n^{2}}{N_{\mathrm{b}, \text { hydro }}}
$$

In turn, $N_{\mathrm{b}, \text { hydro }}$ can be expressed as ${ }^{34}$

$$
N_{\text {b,hydro }}=\frac{C_{\text {b,hydro }} N_{\text {Avo }}}{\mathrm{MW}_{\text {b,hydro }} N_{\mathrm{NPs}}}
$$

where $C_{\mathrm{b} \text {,hydro }}$ is the hydrophilic chain weight concentration in the latex, $\mathrm{MW}_{\mathrm{b} \text {,hydro }}$ is the MW of the hydrophilic chains, $N_{\mathrm{Avo}}$ is the Avogadro number, and $N_{\mathrm{NPs}}$ is the number concentration of NPs in the system. This latter parameter is only a function of the amount of lipophilic blocks according to the hypothesis done above and, in the case of complete conversion, is expressed as ${ }^{35}$

$$
N_{\mathrm{NPs}}=\frac{6 C_{\text {lipo }}}{\pi D n^{3} \rho_{\text {b,lipo }}}
$$

where the $C_{\text {lipo }}$ is the lipophilic monomer weight concentration in the latex and $\rho_{\mathrm{b}, \text { lipo }}$ is the density of the lipophilic chains that form the NP core.

Equations 8, 10, and 11 represent the hypotheses of wellcontrolled living process, hydrophilic blocks located on the NP surface, and lipophilic blocks confined into the core, respectively, and can be combined with eq 9 to obtain the following correlation (detailed derivation in the Supporting Information):

$$
\mathrm{Dn}=\frac{6 \mathrm{DP}_{\text {lipo }} \mathrm{MW}_{\text {lipo }}}{A_{\mathrm{Cov}} N_{\text {Avo }} \rho_{\text {b,lipo }}}
$$

Equation 12 states that the NP Dn is a linear function of the $\mathrm{DP}_{\text {lipo: }}$ the larger the lipophilic part, the bigger the NP. In addition, the $\mathrm{Dn}$ is influenced by the nature of the lipophilic monomer $\left(\mathrm{MW}_{\text {lipo }} / \rho_{\mathrm{b}, \text { lipo }}\right)$ and by the surface area that a hydrophilic chain can cover $\left(A_{\text {cov }}\right)$. Assuming that this equation is true, it is possible to decouple the $\mathrm{DP}_{\text {lipo }}$ from the $\mathrm{Dn}$ if we are able to vary the surface coverage of the hydrophilic chains, e.g., changing the type of the hydrophilic units. When the same hydrophilic unit has to be used, or when a specific binary system has been selected (e.g., polyethylene glycol chains for the synthesis of stealth NPs), the decoupling can be obtained when the $A_{\mathrm{Cov}}$ is a function of the number of hydrophilic units $\left(\mathrm{DP}_{\text {hydro }}\right)$. This can be easily obtained when macro-RAFT surfmers are used, as done in this work and depicted in Figure 1a. In fact, the use of an amphiphilic monomer (PEGMA) leads
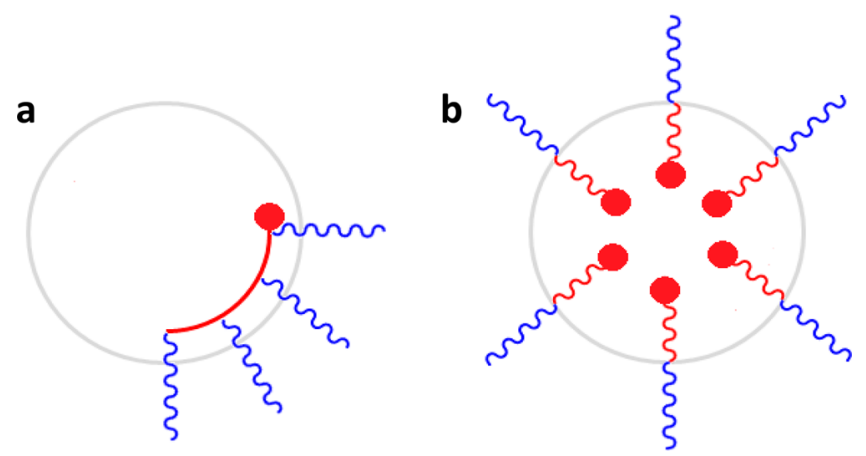

Figure 1. (a) RAFT hydrophilic blocks that derived from monomers that already acts as surfmers. (b) RAFT copolymers usually adopted in the $\mathrm{ab}$ initio RAFT emulsion polymerization.

to a macro-RAFT agent that can be adsorbed onto the NP surface, being the backbone lipophilic (based on polymethacrylate) and the branches hydrophilic (PEO chains). On the contrary, when a block copolymer is used, the surface coverage is only slightly affected by the chain length of the hydrophilic portion, as reported in Figure $1 \mathrm{~b}$.

The variation of the number of the amphiphilic units $\left(\mathrm{DP}_{\text {hydro }}\right)$ added to the macro-RAFT agents adopted in this work can be used to modify the overall ability of these "socalled" RAFT macro-surfmers to cover the NP surface. In addition, since the hydrophilic block has already an amphiphilic nature, it can be directly employed into the AIREP of a lipophilic monomer to synthesize NPs; the procedure requires only two steps, such as the PISA.

Synthesis and Characterization of PEG-Based RAFT Macro-Surfmers. Conversion and $M_{n}$ determined by H NMR can be compared to the ones obtained by GPC in order to verify the assumption about the $\mathrm{d} n / \mathrm{d} c$ and to check qualitatively how good the PS calibration for the evaluation of the RAFT macro-surfmer $M_{\mathrm{n}}$ is.

The kinetics of monomer conversion was also evaluated for all the macro-CTAs. As an example, Figure 2 shows the kinetics data obtained for the polymerization of 20PEGMA. As expected, a logarithmic trend that reaches high conversion at $24 \mathrm{~h}$ of reaction is observed. Table 1 presents the good correspondence between the conversion obtained by GPC and that obtained by NMR, which verifies our assumption about the $\mathrm{d} n / \mathrm{d} c$ of polymer and monomer. A good correspondence can also be observed between the $M_{n}$ obtained by H NMR and the one calculated by eq 1 but not with the one obtained by GPC. This is explained by the use of a PS calibration for the GPC analysis which is not suitable to find precise results in terms of $M_{\mathrm{n}}$. However, the $Ð$ are very low and give us additional proof of the good control over the polymerization process.

CMC Determination of PEG-Based RAFT MacroSurfmers. To evaluate the possibility for PEGylated macroRAFT agents to act as a surface-active species in an emulsion polymerization process, their ability to form polymeric micelles was evaluated in terms of CMC. The fluorescent probe technique was used for the estimation of this parameter using pyrene as the fluorescent probe due to its low solubility in water and photophysical properties. It is well assessed in the 


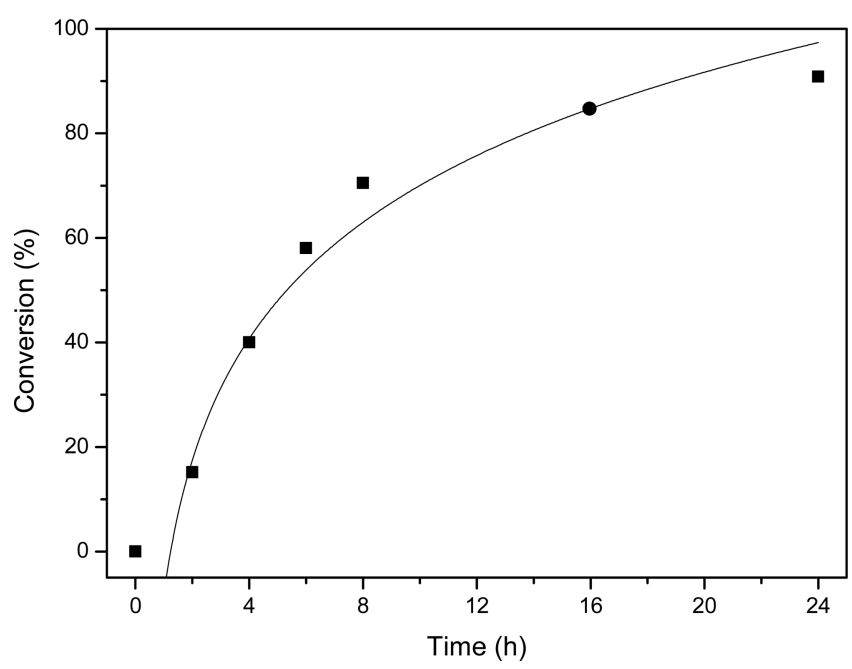

Figure 2. PEGMA conversion as a function of time during the polymerization of 20PEGMA.

literature that the intensity ratio of the pyrene emission peaks at $386 \mathrm{~nm}\left(I_{3}\right)$ and at $373 \mathrm{~nm}\left(I_{1}\right)$ is strongly affected by the nature of the phase in which the pyrene is located. ${ }^{36,37}$ Thus, the study of the $I_{3} / I_{1}$ ratio allows for tracing the pyrene and, in the case of a micellar system, allows to detect the insurgence of a hydrophobic microphase and then to estimate the CMC. Figure 3 reports the $I_{3} / I_{1}$ ratio as a function of the RAFT macro-surfmer concentration.

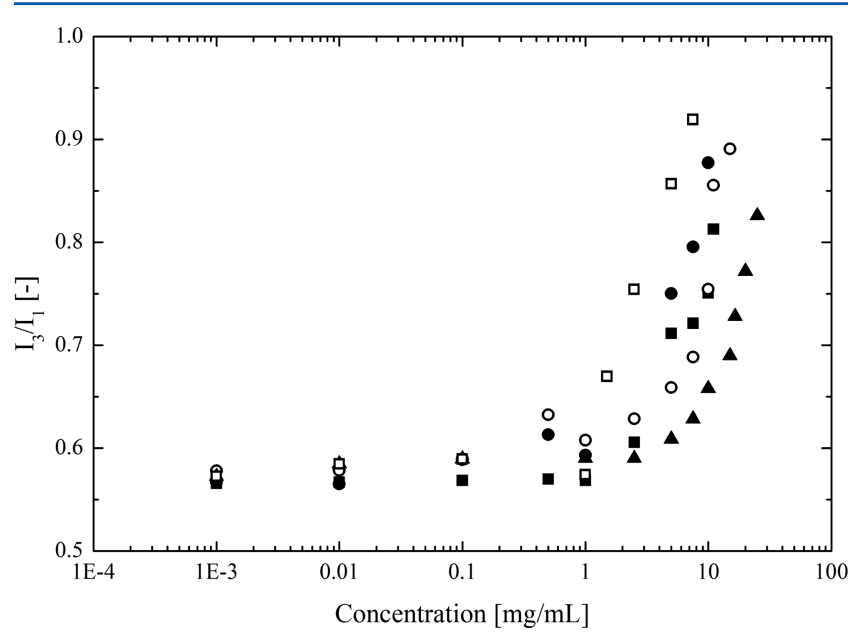

Figure 3. $I_{3} / I_{1}$ ratios as a function of the concentration for PEGMA $(\boldsymbol{\square})$, 5PEGMA ( $\square$ ), 10PEGMA (๑), 20PEGMA (O), and 40PEGMA $(\boldsymbol{\Delta})$ as derived from pyrene emission spectra.

It is possible to observe that the $I_{3} / I_{1}$ ratio assumes an asymptotic value in the range of the low polymer concentrations. This value is approximately equal to 0.57 for all the analyzed samples and is indicative of the presence of the pyrene in an aqueous phase, thus signifying that no micelles were formed. However, when concentrations are increased above the $\mathrm{CMC}$, the pyrene is rapidly stabilized into the newly formed micelles and the $I_{3} / I_{1}$ ratio increases to 0.9 . Figure 3 proves that micellization phenomena occur for both the PEGMA monomer and the four RAFT macro-surfmers synthesized, thus confirming the possibility of using them as surfactants in an emulsion polymerization.
From the concentration dependence of the $I_{3} / I_{1}$ ratio, CMC can be estimated as the intersection of the straight line segments connecting the points lying on a nearly horizontal line and that connecting the points characterized by a rapidly growing $I_{3} / I_{1}$ according to the literature. ${ }^{36,38}$ The CMC values obtained for the PEGMA monomer and for the derived macroRAFT agents are reported in Table 2.

Table 2. CMC Values for PEGMA and PEGMA-Based RAFT Surfmers

\begin{tabular}{lcc} 
sample & CMC $[\mathrm{mg} / \mathrm{mL}]$ & CMC $[\mathrm{mmol} / \mathrm{L}]$ \\
\hline PEGMA & 1.66 & 1.75 \\
5PEGMA & 1.15 & 0.23 \\
10PEGMA & 1.12 & 0.12 \\
20PEGMA & 2.26 & 0.12 \\
40PEGMA & 6.31 & 0.17 \\
\hline
\end{tabular}

While the PEGMA CMC is slightly higher and close to the value already reported in the literature, ${ }^{39}$ the PEG-based RAFT macro-surfmer CMCs values are very similar, highlighting the fact that the MW of the surfmers does not seem to affect their self-assembly behavior, as expected considering that their hydrophilic-lipophilic balance (HLB) is not dependent on the $\mathrm{DP}_{\text {hydro. }}{ }^{40,41}$

NP Synthesis and Characterization. As already mentioned, the aim of this work is to show the possibility of decoupling the dependence between the dimension of the NPs and the MW of the copolymer they are made up of. In order to do that, four different PEGylated RAFT surfmers (5PEGMA, 10PEGMA, 20PEGMA, and 40PEGMA) have been synthesized and used in the ab initio RAFT emulsion polymerization of MMA. As shown in Table 3, monodisperse stable NPs (PDI < 0.2 ) with different $\mathrm{Dn}$ and $M_{\mathrm{n}}$ can be obtained with a conversion higher than $97 \%$ by varying the $\mathrm{DP}_{\text {ratio }}$ from 5 to 20 .

Since the polystyrene calibration has not been able to well describe the $M_{\mathrm{n}}$ of the surfmers, in turn it has not been suited to characterize the final block copolymer $M_{n}$, too. However, "living" conditions has been established, as visible in Figure 4a; the $M_{\mathrm{n}}$ of the block copolymer corresponding to the P5-3 experiment is a linear function of the MMA conversion, and the $Ð$ is always below 1.2. In addition, as shown in Figure $4 \mathrm{~b}$, the $M_{\mathrm{n}}$ is a linear function of the $\mathrm{DP}_{\text {lipo }}$ for the block copolymers within the adoption of the same macro-RAFT surfmer as predicted by eq 8 at complete conversion. However, a small secondary peak is visible and can be ascribed to unreacted macro-RAFT surfmer or dead polymer formed in the production of the macro-RAFT surfmer (Figure S3).

Since the morphology of the NPs are a function of the $\mathrm{DP}_{\text {lipo, }}{ }^{24}$ the TEM has been carried out to verify which type of geometry has been obtained. In all experiments spheres have been produced, as visible in Figure 5.

Decoupling Dn and MW. We have hypothesized that the decoupling of Dn and MW is possible if the surface area that the hydrophilic block of the NP forming copolymer can cover is a function of its length $\left(\mathrm{DP}_{\text {hydro }}\right)$ according to eq 12 . We have also assumed that it is possible to create this dependence by exchanging the hydrophilic block with an amphiphilic one. For this reason, we have synthesized different amphiphilic blocks via RAFT emulsion polymerization of PEGMA, and we have used them in the ab initio RAFT emulsion polymerization of MMA. 
Table 3. Characterization of the NPs Synthesized with Different RAFT Surfmers

\begin{tabular}{|c|c|c|c|c|c|c|c|c|}
\hline $\exp$ & surfmer & $C_{\text {latex }}[\mathrm{wt} \%]$ & $\mathrm{DP}_{\text {lipo }}{ }^{a}$ & $\mathrm{DP}_{\text {ratio }}$ & $M_{\mathrm{n}, \mathrm{GPC}}[\mathrm{g} / \mathrm{mol}]$ & $\bigoplus_{\mathrm{GPC}}$ & $\mathrm{Dn}^{b}[\mathrm{~nm}]$ & PDI \\
\hline P5-1 & SPEGMA & 7 & 25 & 5 & 8495 & 1.18 & 16 & 0.12 \\
\hline P5-2 & 5PEGMA & 9 & 49 & 10 & 10647 & 1.15 & 27 & 0.02 \\
\hline P5-3 & 5PEGMA & 13 & 98 & 20 & 14970 & 1.17 & 51 & 0.05 \\
\hline P10-1 & 10PEGMA & 7 & 47 & 5 & 12179 & 1.23 & 20 & 0.05 \\
\hline P10-2 & 10PEGMA & 9 & 94 & 10 & 14016 & 1.32 & 38 & 0.09 \\
\hline P10-3 & 10PEGMA & 13 & 188 & 20 & 22689 & 1.21 & 67 & 0.15 \\
\hline P20-2 & 20PEGMA & 9 & 189 & 10 & 21146 & 1.28 & 41 & 0.05 \\
\hline P20-3 & 20PEGMA & 13 & 379 & 20 & 30434 & 1.26 & 86 & 0.14 \\
\hline P40-1 & 40PEGMA & 7 & 189 & 5 & 27886 & 1.41 & 30 & 0.13 \\
\hline P40-2 & 40PEGMA & 9 & 378 & 10 & 34065 & 1.27 & 57 & 0.03 \\
\hline P40-3 & 40PEGMA & 13 & 756 & 20 & 54600 & 1.45 & 101 & 0.14 \\
\hline
\end{tabular}

${ }^{a} \mathrm{DP}_{\text {lipo }}=\mathrm{DP}_{\text {ratio }} \cdot \mathrm{DP}_{\text {hydro }}$ where $\mathrm{DP}_{\text {hydro }}$ is the value obtained via ${ }^{1} \mathrm{H}$ NMR and shown in Table $1 .{ }^{b}$ Volume average particle size obtained via DLS.
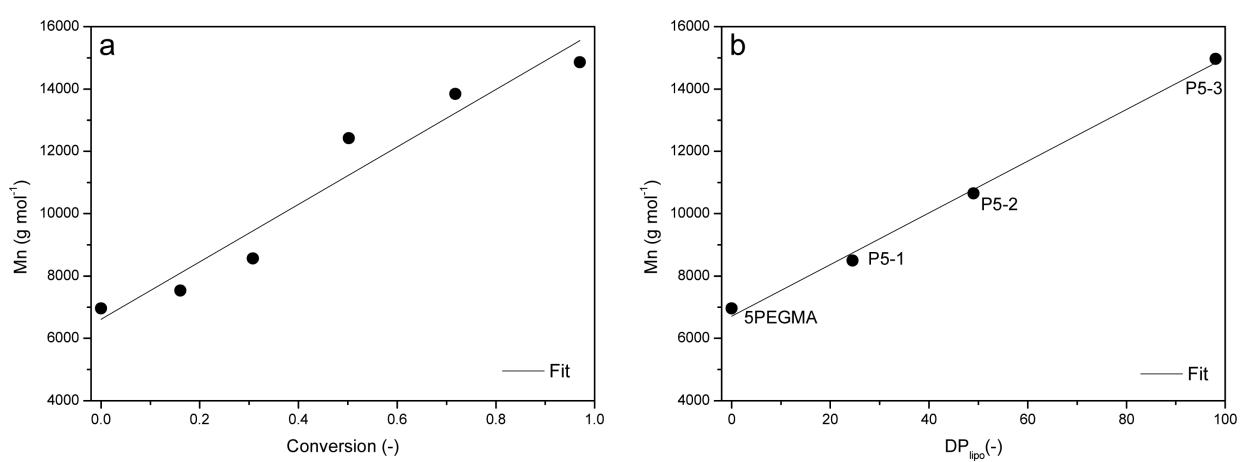

Figure 4. (a) $M_{\mathrm{n}}$ as a function of MMA conversion for the block copolymer corresponding to entry P5-3. (b) $M_{\mathrm{n}}$ as a function of DP lipo for block copolymers corresponding to entries P5-1, P5-2, and P5-3 in Table 3.
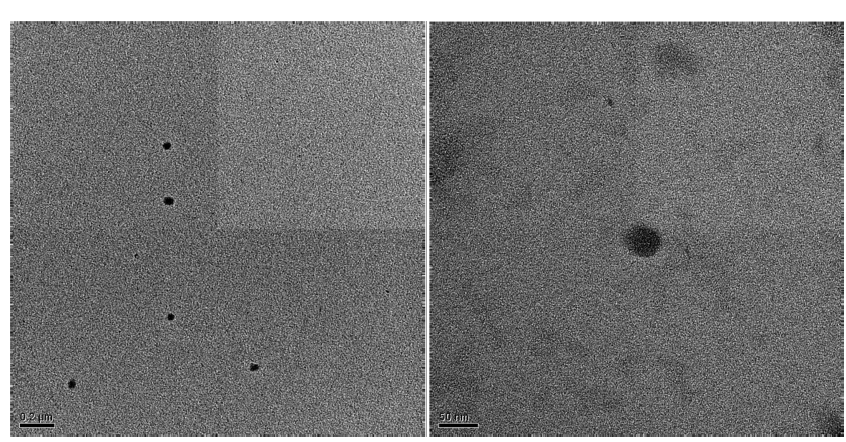

Figure 5. TEM images of the NPs obtained in the P5-3 experiment in Table 3.

We have also verified (i) the validity of the eq 8 and of the well-controlled RAFT polymerizations by the production of well-defined block copolymers and (ii) the spherical geometry of the obtained NPs via TEM analysis.

In order to verify the possibility of the decoupling, eq 12 has been used to evaluate the $A_{\text {cov }}$ for the experiments in Table 3 with $\mathrm{DP}_{\text {ratio }}=10$ according to

$$
A_{\mathrm{Cov}}=\frac{6 \cdot \mathrm{DP}_{\text {lipo }} \cdot \mathrm{MW}_{\mathrm{MMA}}}{\mathrm{Dn} \cdot N_{\mathrm{Avo}} \cdot \rho_{\mathrm{b}, \mathrm{lipo}}}
$$

As visible in Figure 6, the $A_{\text {cov }}$ is clearly a linear function of the $\mathrm{DP}_{\text {hydro, }}$ and thus, it confirms our assumption that higher numbers of units of the RAFT macro-surfmer correspond to improved abilities to cover more of the NP surface. It is noteworthy important to mention that the use of Dn obtained

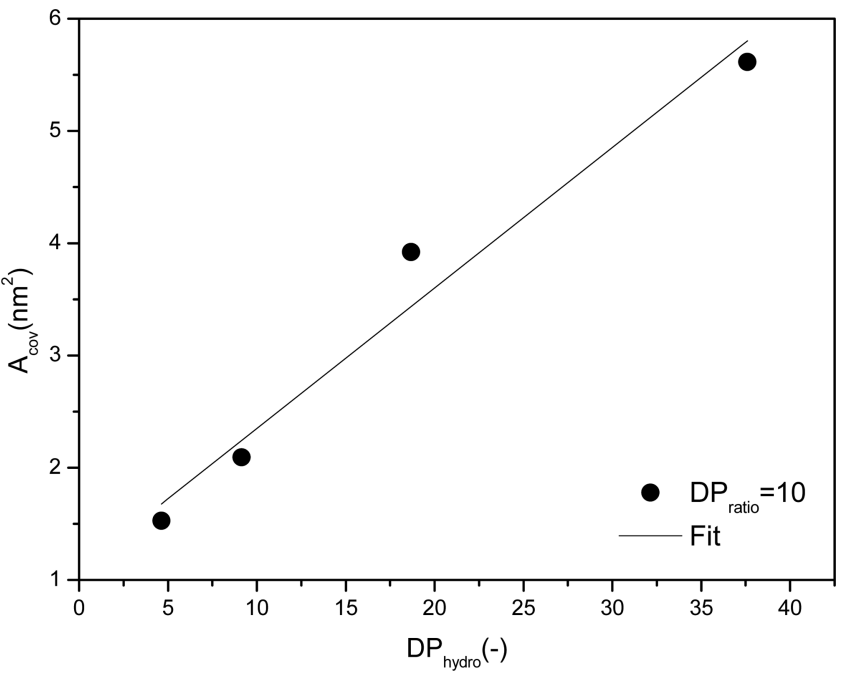

Figure 6. $A_{\text {cov }}$ as a function of $\mathrm{DP}_{\text {hydro }}$ evaluated for all data with $\mathrm{DP}_{\text {ratio }}$ $=10$ in Table 3 according to eq 13 .

via DLS in this equation implies that the contribution of the hydrophilic chains to the NP hydrodynamic volume is negligible. This approximation is reasonable when very short hydrophilic chains are responsible for the NP stabilization, such as the 19 ethylene glycol units of the PEGMA that contribute to the final NP size by less than $5 \mathrm{~nm}$, as reported in the literature. $^{22,42}$

To better visualize the implication of these findings, this linear dependence has been fitted and substituted into the same 

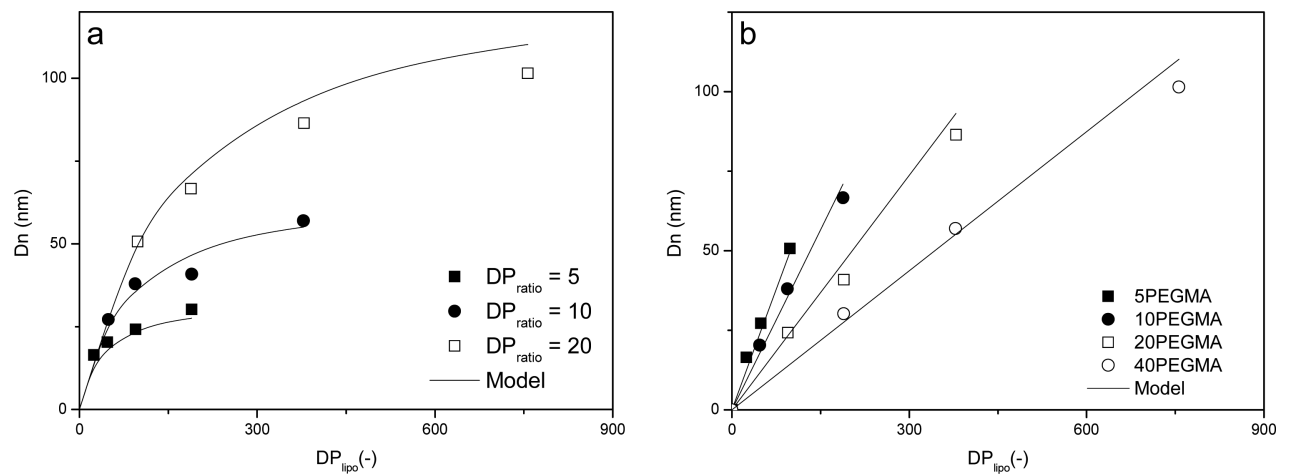

Figure 7. (a) Data simulations through eq 13 at different $\mathrm{DP}_{\text {ratio }}$ and experimental values. (b) Data simulations through eq 13 at different $\mathrm{DP}_{\text {hydro }}$ and experimental values.
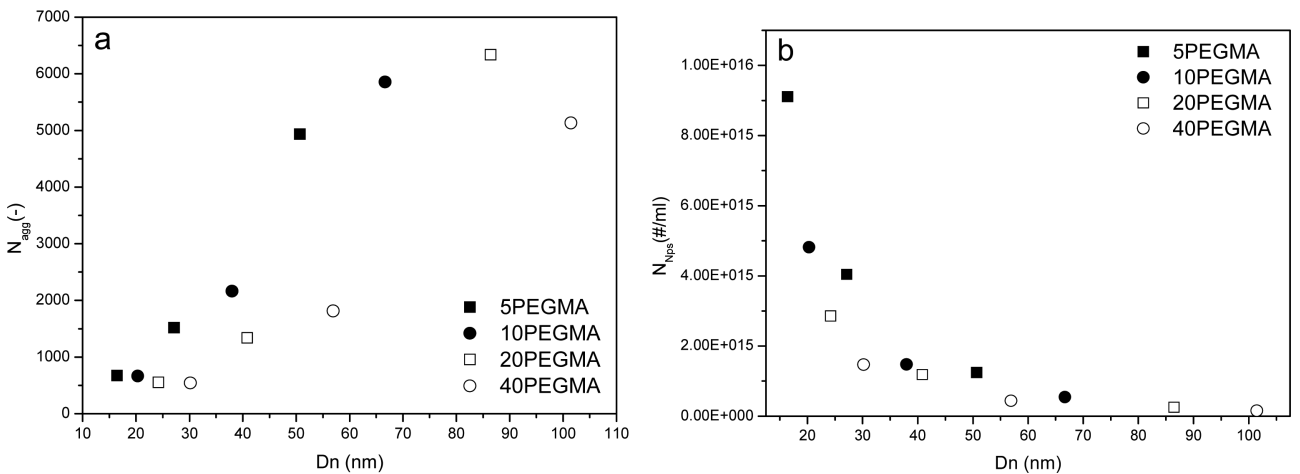

Figure 8. (a) $N_{\text {agg }}$ as a function of $\mathrm{Dn}$ at different $\mathrm{DP}_{\text {hydro }}$ evaluated according to eq 15. (b) NP numeric concentration as a function of Dn evaluated according to eq 11 .

eq 13 that we have used to derive it with the data at $\mathrm{DP}_{\text {ratio }}=10$ according to

$$
\mathrm{Dn}=\frac{6 \mathrm{DP}_{\text {lipo }} \mathrm{MW}_{\text {lipo }}}{\left(\alpha \mathrm{DP}_{\text {hydro }}+\beta\right) N_{\text {Avo }} \rho_{\text {b,lipo }}}
$$

Then we have adopted the new eq 14 to simulate the dependence of the $\mathrm{Dn}$ from the $\mathrm{DP}_{\text {lipo }}$ at the same $\mathrm{DP}_{\text {ratio }}$ and compared with all the experimental data, as visible in Figure $7 \mathrm{a}$. In the case of the samples at $\mathrm{DP}_{\text {ratio }}=10$, the simulation is only the description of how good the fitting is. Otherwise, it is predictive and also in good agreement for the experiments at $\mathrm{DP}_{\text {ratio }}=5$ and 20. Indeed, it shows the possibility of obtaining the desired $\mathrm{Dn}$ and length of the lipophilic block $\left(\mathrm{DP}_{\text {lipo }}\right)$ that forms the NP by adopting the RAFT macro-surfmer with the correct length $\left(\mathrm{DP}_{\text {hydro }}\right)$. In addition, eq 14 is able to predict the linear dependence of the $\mathrm{Dn}$ from the $\mathrm{DP}_{\text {lipo }}$ at the same $\mathrm{DP}_{\text {hydro, }}$ as visible in Figure $7 \mathrm{~b}$. The slopes of these linear functions are related to $A_{\text {cov }}$ and are constant within the adoption of the same macro-RAFT surfmer. A similar linear dependence of $\mathrm{Dn}$ from $\mathrm{DP}_{\text {lipo }}$ is also visible in the literature in other works where different hydrophilic blocks have been adopted to stabilize latex of different lipophilic monomers. ${ }^{34,35,43,44}$ As an example, in the Zhang et al. work ${ }^{34}$ two hydrophilic poly(methacrylic acid-co-poly(ethylene oxide) methyl ether methacrylate) RAFT surfmers of different compositions have been synthesized and adopted in the RAFT emulsion polymerization of styrene. In this case, the $A_{\text {cov }}$ for the two systems have been evaluated, and their values are comparable to those obtained in our study. All these results seem to indicate that $A_{\text {cov }}$ is an intrinsic property of the stabilizing part of the block copolymer and that eq 13 has a more general validity for the block copolymer nanoparticles under the assumption made above. In fact, the linear behavior indicates that $A_{\text {cov }}$ within the adoption of the same lipophilic monomer is not a function of the length of the lipophilic chain. In addition, as shown in several works, ${ }^{35,45}$ the NPs seems to grow linearly with the extension of the lipophilic block and, thus, with the overall monomer conversion. In fact, as the RAFT emulsion is a "living" polymerization, the overall molecular weight is a linear function of the conversion according to eq 8 .

In addition, the slope of the linear functions in Figure $7 \mathrm{~b}$ decreases with the increase of the $A_{\text {cov }}$ of the amphiphilic block and, thus, of the increase of $\mathrm{DP}_{\text {hydro }}$; to obtain the same $\mathrm{Dn}$, it is necessary to add more MMA units if the length of the RAFT macro-surfmer is bigger. This phenomenon can be understood by recalling the assumptions that the lipophilic chains and the amphiphilic ones are mainly located in the core and on the surface, respectively. Since their numbers are equal because they form the same block copolymer, fewer units of hydrophilic chains on the surface correspond to lower lipophilic chains in the core, and thus, more MMA units for each chain $\left(\mathrm{DP}_{\text {lipo }}\right)$ will be necessary to fill the volume of the NP core.

The phenomenon is more clear if we consider the number of aggregation $\left(N_{\mathrm{agg}}\right)$, the number of chains for each $\mathrm{NP}$, according to ${ }^{35}$

$$
N_{\text {agg }}=\frac{\pi \mathrm{Dn}}{6 \mathrm{DP}_{\text {lipo }} \mathrm{MW}_{\text {lipo }}}
$$

where only the pMMA chains are considered to be involved in the formation of the NP core. 
In fact, the higher the number of chains $\left(N_{\mathrm{agg}}\right)$ required to form a NP with the same $\mathrm{Dn}$, the smaller the $\mathrm{DP}_{\text {hydro }}$ as visible in Figure 8a; at the same time the chains are shorter, as already seen in Figure $7 \mathrm{~b}$.

Since we adopted the same concentration of macro-RAFT surfmer for each experiment, the reduction of the $N_{\mathrm{NPs}}$, as visible in Figure $8 \mathrm{~b}$, indicates that a migration of block copolymer to the growing NP surface or diassembly/assembly phenomena of NPs have occurred during the RAFT emulsion polymerization allowing the coverage and stabilization of the forming surface of the growing NPs. ${ }^{34,35}$ As previously reported in the section of the CMC evaluation, the amphiphilic nature of the PEG-based macro-RAFT surfmers is not a function of their lengths due to the disposition of the PEG chains depicted in Figure 1a, but it is related to the intrinsic ability of the 19 units of ethylen glycol to act as steric stabilizer. If a fixed set of PEG branches are required to stabilize a certain NP surface area, this number is ideally not affected by how many subsets it is divided into $\left(\mathrm{DP}_{\text {hydro }}\right)$. For this reason, the $N_{\mathrm{NPs}}$ is not significantly influenced by the $\mathrm{DP}_{\text {hydro }}$, as demonstrated in Figure $8 \mathrm{~b}$.

Equation 13 can be used not only to independently tune the length of the lipophilic chains and the NP Dn by varying the length of the amphiphilic block but also to decouple the dependence between the Dn and the MW of the whole block copolymer $\left(\mathrm{MW}_{\text {cop }}\right)$ by solving eqs 8 and 12 at the same time. However, we expect that some physical and geometric conditions will limit our decoupling possibility. In order to find them, a 3-D simulation has been carried out in Matlab. As visible in Figure 9, it is possible to choose the desired $\mathbf{M W}_{\text {cop }}$

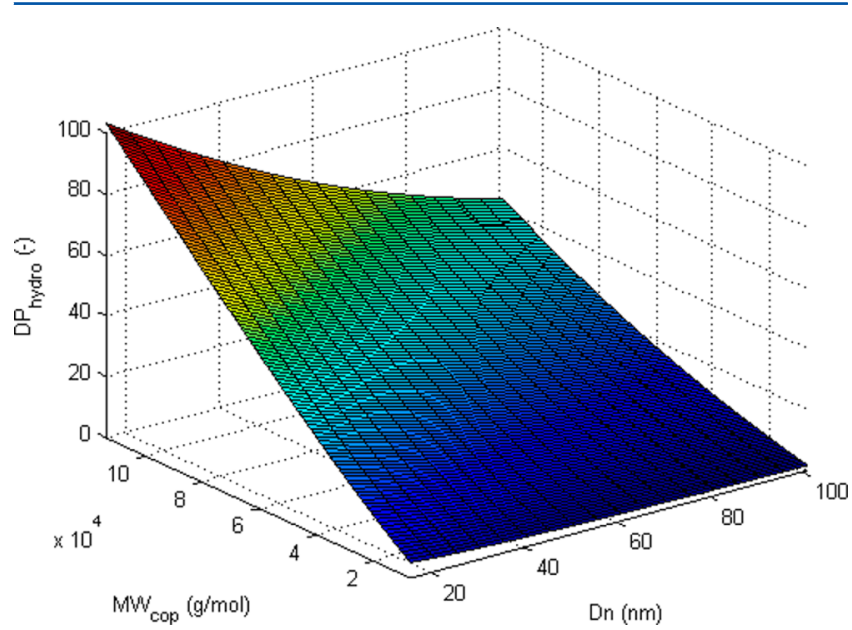

Figure 9. Solutions of eqs 8 and 12: $\mathrm{DP}_{\text {hydro }}$ as a function of Dn and $\mathrm{MW}_{\text {cop. }}$.

and $\mathrm{Dn}$ and find which $\mathrm{DP}_{\text {hydro }}$ is necessary to obtain them. Then the $\mathrm{DP}_{\text {lipo }}$ can be immediately evaluated by eq 8 . With this method, it is possible to obtain a variety of $\mathrm{Dn}$ and $\mathrm{MW}_{\text {cop }}$ and even very small NPs with very long block copolymers. However, the production of big NPs with very short block copolymer is not possible, and this is consistent with the geometric and physical constraints of the system: the lipophilic blocks have to be long and/or numerous enough to fill the volume of the NP core.

\section{CONCLUSION}

In this work the theoretical conditions necessary to decouple the NP Dn and MW in the ab initio RAFT emulsion polymerization have been developed. It has been found that it is possible by adopting the so-called RAFT macro-surfmers: RAFT macromolecular amphipilic species produced by the RAFT polymerization of a surfmer. Their use allows for a substitution of the hydrophilic chains of the final block copolymer formed via AIREP with amphiphilic chains. To prove this possibility and show the procedure, we have synthesized well-defined block copolymers that consist of an amphiphilic part and a lipophilic one via a two-step procedure: (i) the RAFT polymerization of a PEGylated species that has an intrinsic amphiphilic nature (PEGMA) and (ii) the ab initio RAFT emulsion polymerization of MMA in the presence of this RAFT macro-surfmer. The $A_{\text {cov }}$ of these new amphiphilic species has been found to be a function of their length and have been allowed to synthesize monodisperse NPs with different sizes and MW. By adopting the correct length of the amphiphilic block, it has been proven possible to obtain the desired length of the lipophilic block or the MW of the whole block copolymer and the desired NP. It has been shown that with this procedure it is not possible to produce very big NPs with very low MW, a finding that is consistent with the physical and geometric constraints of the system. As long as the theoretical hypotheses under the possibility to tune MW and Dn independently are obtained mainly from geometrical consideration and from the assumption of well-controlled RAFT polymerization, it is expected that also the nanoprecipitation of block copolymers made with the same amphiphilic part will have a similar behavior. Further studies will be necessary to confirm and clarify the implication of these findings.

\section{ASSOCIATED CONTENT}

\section{S Supporting Information}

The Supporting Information is available free of charge on the ACS Publications website at DOI: 10.1021/acs.macromol.6b01827.

${ }^{1} \mathrm{H}$ NMR spectrum of the sample 20PEGMA after $24 \mathrm{~h}$ of reaction; GPC trace of the sample 20PEGMA after 24 $\mathrm{h}$ of reaction; GPC traces of the block copolymers produced with 5PEGMA in the AIREP experiments P51, P5-2, and P5-3 in Table 3; list of abbreviations; derivation of eq 12 from eqs $8-11$ (PDF)

\section{AUTHOR INFORMATION}

\section{Corresponding Author}

*(D.M.) E-mail davide.moscatelli@polimi.it. ORCID

Davide Moscatelli: 0000-0003-2759-9781

\section{Notes}

The authors declare no competing financial interest.

\section{REFERENCES}

(1) Braunecker, W. A.; Matyjaszewski, K. Controlled/living radical polymerization: Features, developments, and perspectives. Prog. Polym. Sci. 2007, 32 (1), 93-146.

(2) Sun, J.-T.; Hong, C.-Y.; Pan, C.-Y. Recent advances in RAFT dispersion polymerization for preparation of block copolymer aggregates. Polym. Chem. 2013, 4 (4), 873-881.

(3) Cunningham, M. F. Controlled/living radical polymerization in aqueous dispersed systems. Prog. Polym. Sci. 2008, 33 (4), 365-398. 
(4) Zetterlund, P. B.; Thickett, S. C.; Perrier, S.; Bourgeat-Lami, E.; Lansalot, M. Controlled/Living Radical Polymerization in Dispersed Systems: An Update. Chem. Rev. 2015, 115 (18), 9745-9800.

(5) Li, Y.; Armes, S. P. RAFT synthesis of sterically stabilized methacrylic nanolatexes and vesicles by aqueous dispersion polymerization. Angew. Chem., Int. Ed. 2010, 49 (24), 4042-4046.

(6) Steward, P. A.; Hearn, J.; Wilkinson, M. C. An overview of polymer latex film formation and properties. Adv. Colloid Interface Sci. 2000, 86 (3), 195-267.

(7) do Amaral, M.; Roos, A.; Asua, J. M.; Creton, C. Assessing the effect of latex particle size and distribution on the rheological and adhesive properties of model waterborne acrylic pressure-sensitive adhesives films. J. Colloid Interface Sci. 2005, 281 (2), 325-338.

(8) Brannon-Peppas, L.; Blanchette, J. O. Nanoparticle and targeted systems for cancer therapy. Adv. Drug Delivery Rev. 2012, 64, 206-212.

(9) Owens, D. E.; Peppas, N. A. Opsonization, biodistribution, and pharmacokinetics of polymeric nanoparticles. Int. J. Pharm. 2006, 307, 93-102.

(10) Elsabahy, M.; Wooley, K. L. Design of polymeric nanoparticles for biomedical delivery applications. Chem. Soc. Rev. 2012, 41 (7), $2545-2561$.

(11) Nomura, M.; Tobita, H.; Suzuki, K. Emulsion polymerization: Kinetic and mechanistic aspects. In Polymer Particles; Springer: 2005; pp $1-128$.

(12) Chern, C. S. Emulsion polymerization mechanisms and kinetics. Prog. Polym. Sci. 2006, 31 (5), 443-486.

(13) Sajjadi, S. Nanoparticle formation by monomer-starved semibatch emulsion polymerization. Langmuir 2007, 23 (3), 10181024

(14) Cserháti, T.; Forgács, E.; Oros, G. Biological activity and environmental impact of anionic surfactants. Environ. Int. 2002, 28 (5), 337-348.

(15) Ying, G.-G. Fate, behavior and effects of surfactants and their degradation products in the environment. Environ. Int. 2006, 32 (3), 417-431.

(16) Hawker, C. J.; Bosman, A. W.; Harth, E. New polymer synthesis by nitroxide mediated living radical polymerizations. Chem. Rev. 2001, 101 (12), 3661-3688.

(17) Wang, J.-S.; Matyjaszewski, K. Controlled/“living” radical polymerization. Atom transfer radical polymerization in the presence of transition-metal complexes. J. Am. Chem. Soc. 1995, 117 (20), 5614-5615.

(18) Chiefari, J.; Chong, Y.; Ercole, F.; Krstina, J.; Jeffery, J.; Le, T. P.; Mayadunne, R. T.; Meijs, G. F.; Moad, C. L.; Moad, G. Living freeradical polymerization by reversible addition-fragmentation chain transfer: the RAFT process. Macromolecules 1998, 31 (16), 55595562 .

(19) Li, Y.; Schadler, L.; Benicewicz, B.; Barner-Kowollik, C. Handbook of RAFT Polymerization; Wiley-VCH: Weinheim, 2008.

(20) Chong, Y.; Le, T. P.; Moad, G.; Rizzardo, E.; Thang, S. H. A more versatile route to block copolymers and other polymers of complex architecture by living radical polymerization: the RAFT process. Macromolecules 1999, 32 (6), 2071-2074.

(21) Butté, A.; Storti, G.; Morbidelli, M. Miniemulsion living free radical polymerization by RAFT. Macromolecules 2001, 34 (17), $5885-5896$.

(22) Colombo, C.; Gatti, S.; Ferrari, R.; Casalini, T.; Cuccato, D.; Morosi, L.; Zucchetti, M.; Moscatelli, D. Self-assembling amphiphilic PEGylated block copolymers obtained through RAFT polymerization for drug-delivery applications. J. Appl. Polym. Sci. 2016, 133 (11), 43084 .

(23) Ferguson, C. J.; Hughes, R. J.; Nguyen, D.; Pham, B. T.; Gilbert, R. G.; Serelis, A. K.; Such, C. H.; Hawkett, B. S. Ab Initio Emulsion Polymerization by RAFT-Controlled Self-Assembly. Macromolecules 2005, 38 (6), 2191-2204.

(24) Rieger, J. Guidelines for the Synthesis of Block Copolymer Particles of Various Morphologies by RAFT Dispersion Polymerization. Macromol. Rapid Commun. 2015, 36 (16), 1458-1471.
(25) Sebakhy, K. O.; Kessel, S.; Monteiro, M. J. Nanoreactors to synthesize well-defined polymer nanoparticles: decoupling particle size from molecular weight. Macromolecules 2010, 43 (23), 9598-9600.

(26) Charleux, B.; Delaittre, G.; Rieger, J.; D’Agosto, F. Polymerization-induced self-assembly: from soluble macromolecules to block copolymer nano-objects in one step. Macromolecules 2012, 45 (17), 6753-6765.

(27) Chaduc, I.; Girod, M.; Antoine, R.; Charleux, B.; D’Agosto, F.; Lansalot, M. Batch Emulsion Polymerization Mediated by Poly(methacrylic acid) MacroRAFT Agents: One-Pot Synthesis of SelfStabilized Particles. Macromolecules 2012, 45 (15), 5881-5893.

(28) Zhou, J.; Wang, H.; Zhang, L.; Ma, J. Ab initio reversible addition-fragmentation chain transfer emulsion polymerization of styrene/butyl acrylate mediated by poly(acrylic acid)-block-polystyrene trithiocarbonate. Polym. Int. 2014, 63 (12), 2098-2104.

(29) Huang, J.; Zhao, S.; Gao, X.; Luo, Y.; Li, B. RAFT Ab Initio Emulsion Polymerization of Styrene Using Poly(acrylic acid)-bpolystyrene Trithiocarbonate of Various Structures as Mediator and Surfactant. Macromol. React. Eng. 2014, 8 (10), 696-705.

(30) Zhu, Y.; Bi, S.; Gao, X.; Luo, Y. Comparison of RAFT Ab Initio Emulsion Polymerization of Methyl Methacrylate and Styrene Mediated by Oligo(methacrylic acid-b-methyl methacrylate) Trithiocarbonate Surfactant. Macromol. React. Eng. 2015, 9 (5), 503-511.

(31) Zhou, J.; Zhang, L.; Ma, J. Fluorinated polyacrylate emulsifierfree emulsion mediated by poly(acrylic acid)-b-poly(hexafluorobutyl acrylate) trithiocarbonate via ab initio RAFT emulsion polymerization. Chem. Eng. J. 2013, 223, 8-17.

(32) Ganeva, D. E.; Sprong, E.; de Bruyn, H.; Warr, G. G.; Such, C. H.; Hawkett, B. S. Particle Formation in ab Initio RAFT Mediated Emulsion Polymerization Systems. Macromolecules 2007, 40 (17), 6181-6189.

(33) Asua, J.; Schoonbrood, H. Reactive surfactants in heterophase polymerization. Acta Polym. 1998, 49 (12), 671-686.

(34) Zhang, W.; D’Agosto, F.; Boyron, O.; Rieger, J.; Charleux, B. One-pot synthesis of poly (methacrylic acid-co-poly (ethylene oxide) methyl ether methacrylate)-b-polystyrene amphiphilic block copolymers and their self-assemblies in water via RAFT-mediated radical emulsion polymerization. A kinetic study. Macromolecules 2011, 44 (19), 7584-7593.

(35) Su, Y.; Xiao, X.; Li, S.; Dan, M.; Wang, X.; Zhang, W. Precise evaluation of the block copolymer nanoparticle growth in polymerization-induced self-assembly under dispersion conditions. Polym. Chem. 2014, 5 (2), 578-587.

(36) Astafieva, I.; Zhong, X. F.; Eisenberg, A. Critical Micellization Phenomena in Block Polyelectrolyte Solutions. Macromolecules 1993, 26 (26), 7339-7352.

(37) Zhao, C. L.; Winnik, M. A.; Riess, G.; Croucher, M. D. Fluorescence Probe Techniques Used to Study Micelle Formation in Water-Soluble Block Copolymers. Langmuir 1990, 6 (2), 514-516.

(38) Li, S. S.; Liu, Y.; Ji, S.; Zhou, Z.; Li, Q. F. Synthesis and selfassembly behavior of thermoresponsive poly(oligo(ethylene glycol) methyl ether methacrylate)-POSS with tunable lower critical solution temperature. Colloid Polym. Sci. 2014, 292 (11), 2993-3001.

(39) Mirmira, P.; Evans-Molina, C.; Bisphenol, A. obesity, and type 2 diabetes mellitus: genuine concern or unnecessary preoccupation? Transl. Res. 2014, 164 (1), 13-21.

(40) Griffin, W. C. Classification of surface-active agents by "HLB". J. Soc. Cosmet. Chem. 1946, 1, 311-326.

(41) Griffin, W. C. Calculation of HLB values of non-ionic surfactants. Am. Perfumer Essent Oil Rev. 1955, 65, 26-29.

(42) Ferrari, R.; Colombo, C.; Casali, C.; Lupi, M.; Ubezio, P.; Falcetta, F.; D'Incalci, M.; Morbidelli, M.; Moscatelli, D. Synthesis of surfactant free PCL-PEG brushed nanoparticles with tunable degradation kinetics. Int. J. Pharm. 2013, 453 (2), 551-559.

(43) Rieger, J.; Stoffelbach, F.; Bui, C.; Alaimo, D.; Jérôme, C.; Charleux, B. Amphiphilic poly (ethylene oxide) macromolecular RAFT agent as a stabilizer and control agent in ab initio batch emulsion polymerization. Macromolecules 2008, 41 (12), 4065-4068. 
(44) Jones, E.; Mykhaylyk, O.; Semsarilar, M.; Boerakker, M.; Wyman, P.; Armes, S. How Do Spherical Diblock Copolymer Nanoparticles Grow during RAFT Alcoholic Dispersion Polymerization? Macromolecules 2015, 49 (1), 172-181.

(45) Xiao, X.; He, S.; Dan, M.; Su, Y.; Huo, F.; Zhang, W. Brush macro-RAFT agent mediated dispersion polymerization of styrene in the alcohol/water mixture. J. Polym. Sci., Part A: Polym. Chem. 2013, 51 (15), 3177-3190. 